\title{
Imaging of Bacterial External Polymeric Substances by Methods of SEM
}

Bruce W. Arey*, Alexander Laskin* and Alice Dohnalkova*

*W. R. Wiley Environmental Molecular Sciences Laboratory, Pacific Northwest National Laboratory, P.O. Box 999, K8-93, Richland, WA 99354

External polymeric substances (ExPS) play an important role in processes that govern microbial adsorption to cells and surfaces, biofilm formation, and also influence geochemical processes by serving as sites of microbial metal binding [1]. This category includes lipopolysacharides (LPS) and exopolysacharides (EPS) and several other substances that have a common feature of water-binding hydrocarbons with repeating units of periodically linked sugars and, respectively, lipids. All of these structures have general characteristics of hydrated, very thin films of branched, veil-like material associated with and surrounding the cells. ExPS also carry patches of negative and positive charged areas. [2].

Because of their physical properties, ExPS undergo dramatic morphological changes during sample preparation for electron microscopy. During the dehydration, cells collapse into arrangements that do not reflect their native state. Since the attention is usually focused on the bacterial cells rather than the ExPS, methods such as critical point drying (CPD) that prevent the cells collapse are successfully used. However, from our observations, the ExPS has a tendency to condense into stringy structures that do not reflect the native appearance. The images of ExSP obtained after high pressure freezing and imaging cells in the cryo SEM represent, to our belief, the natural situation the most accurately. This method is superior to other methods for stabilization of ExPS such as incubation with Alcian blue, ruthenium red, or charged nanogold. We were looking into a method that could be used with a conventional SEM, and would not require highly specialized instrumentation and expertise. Here we report on the use of an environmental cell for imaging wet samples in a conventional high vacuum SEM.

QuantomiX QX-102 capsule technology presents innovative approach in imaging of wet samples applied to a very thin membrane that is electron-transparent, and allows imaging in a back-scattered or in-lens mode. A small volume of sample is applied in a liquid dish $(3 \mathrm{~mm}$ in diameter), liquid replaced with a buffer, and then aspirated. The capsule is closed and transferred to the SEM. Imaging is done in a low or high vacuum in $10-30 \mathrm{kV}$. The method of sample preparation is very quick and simple, and the specimen does not need to be sputtercoated with a metal to prevent charging.

QuantomiX cell enabled us to study hydrated samples of bacterial suspension in a conventional SEM. Bacterial cells of Shewanella oneidensis MR-1, a rod-shaped soil bacterium, were stained with uranyl acetate and washed. A $15 \mu \mathrm{l}$ drop of cell suspension was applied to the capsule's liquid dish, volume of $\sim 10 \mu 1$ was withdrawn, and the cell was closed. FESEM LEO 982 was used for imaging in backscattered and in-lens mode at $20 \mathrm{kV}$. As anticipated, we were able to distinguish a delicate spongy structure of ExPS, in contrast with a rigid stringy condensed structures observed in samples prepared by the critical point drying. On the other hand, due to the nature of this method, one can not observe fine cellular features such as 
flagella and pili. This did not concern us because these structures can be imaged by different means of EM, and since they are not hydrated, their structure does not suffer by a conventional dehydration.

\section{References}

[1] Parker D.L. et al., J. Appl. Phycology 12: 219-224 (2000).

[2] Lins R.D. and Straatsma T.P., Biophysical Journal 81: 1037-1046 (2001)

This work was conducted at the W. R. Wiley Environmental Molecular Sciences Laboratory (EMSL), multiprogram national laboratory operated by Battelle for the U.S. DOE under Contract DE-AC05-76RLO1830.

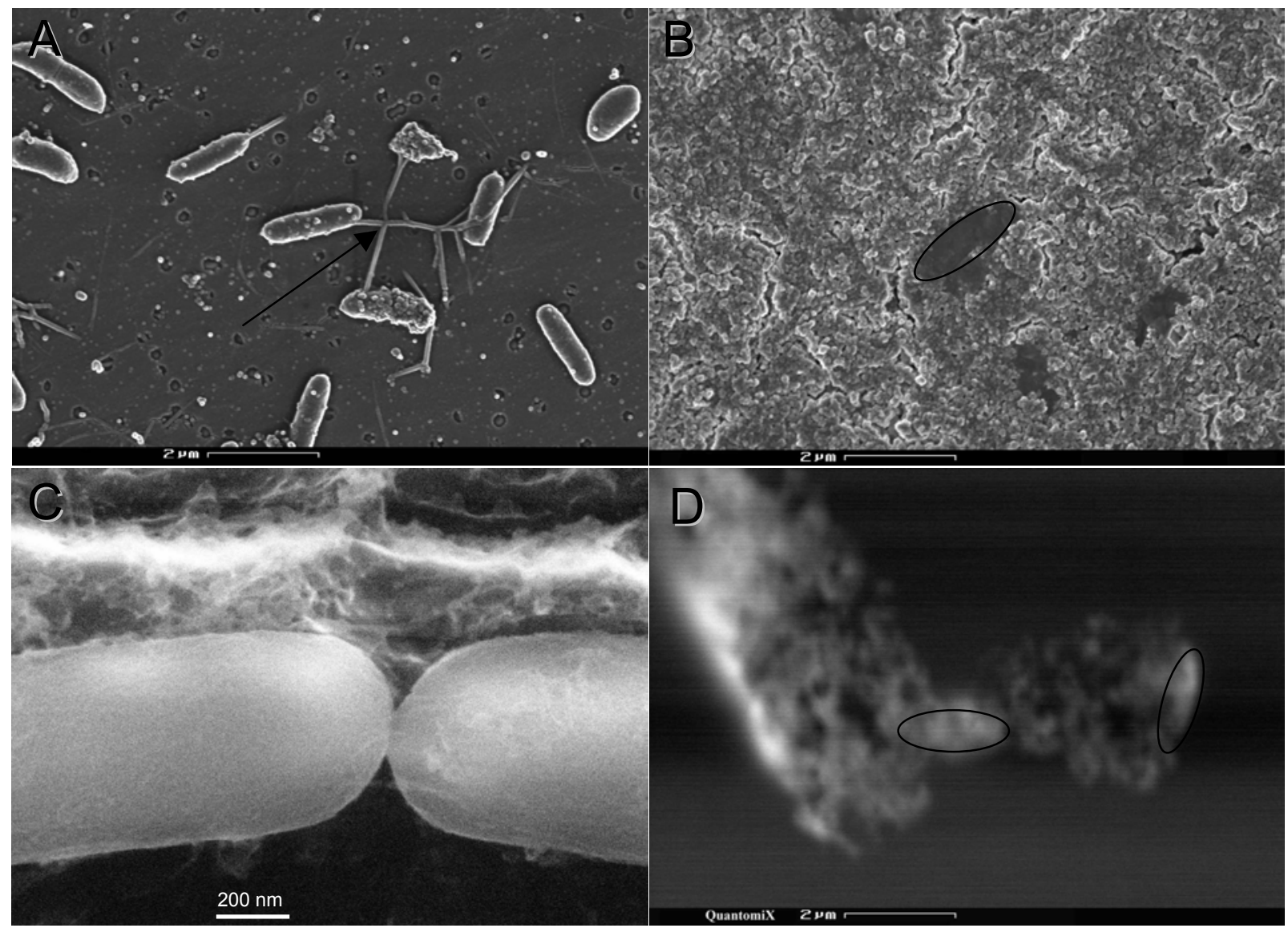

Fig.1. SEM image of a Shewanella suspension. (A) prepared by CPD. Notice the strands of ExPS induced by dehydration and CPD processing (arrow). (B) prepared by air drying. The precipitates of salts contained in buffer crystallized on EPS surface, and interfered with cell structure. (C) Cryo-SEM of Shewanella showing the veil-like structure of ExPS. (D) QuantomiX wet cell preparation preserves the EPS in its natural hydrated state. Oval shape cells structure appears in (B) and (D) are encrusted with the media salts, and covered by the ExPS, respectively. 